Pacific

Journal of

Mathematics

LIMIT THEOREM FOR INVERSE SEQUENCES OF METRIC SPACES IN EXTENSION THEORY

Leonard R. Rubin and Philip J. Schapiro 


\title{
LIMIT THEOREM FOR INVERSE SEQUENCES OF METRIC SPACES IN EXTENSION THEORY
}

\author{
LeOnard R. Rubin And Philip J. Schapiro
}

\begin{abstract}
We prove a limit theorem for extension theory for metric spaces. This theorem can be put in the following way. Suppose that $K$ is a simplicial complex, $|K|$ is given the weak topology, and a metrizable space $X$ is the limit of an inverse sequence of metrizable spaces $X_{i}$ having the property that $X_{i} \tau|K|$ for each $i \in \mathbb{N}$. Then $X \tau|K|$. This latter property means that for each closed subset $A$ of $X$ and map $f: A \rightarrow|K|$, there exists a map $F: X \rightarrow|K|$ which is an extension of $f$.

As a corollary to this we get the result of Nagami that the limit of an inverse sequence of metrizable spaces each having dimension $\leq n$ has dimension $\leq n$. But we get much more, as this result extends to cohomological dimension modulo an abelian group. Hence, if $G$ is an abelian group and $X$ is the limit of an inverse sequence of metrizable spaces $X_{i}$ where $\operatorname{dim}_{G} X_{i} \leq n$ for each $i \in \mathbb{N}$, then $\operatorname{dim}_{G} X \leq n$.
\end{abstract}

\section{Introduction.}

In this paper we are going to prove a limit theorem for extension theory in arbitrary metrizable spaces. The theorem goes as follows. Let $K$ be a simplicial complex and $|K|$ have the weak topology. Suppose that $X$ is the limit of an inverse sequence of metrizable spaces $X_{i}$ where for each $i \in \mathbb{N}$, $X_{i} \tau|K|$. Then $X \tau|K|$. This latter notation means that for each closed subset $A$ of $X$ and map $f: A \rightarrow|K|$, there exists a map $F: X \rightarrow|K|$ which is an extension of $f$.

If in place of $|K|$ we put $S^{n}$, then $X \tau|K|$ means that $\operatorname{dim} X \leq n$. Similarly, for an abelian group $G, X \tau K(G, n)$ means that $\operatorname{dim}_{G} X \leq n$. This idea of treating dimension theory as a branch of extension theory is not new, although it recently has received a lot of play, particularly in the works of Dranishnikov and Dydak, e.g., [DD]. Indeed, the paper [Wa] of John Walsh in which the Edwards-Walsh theorem is proved could be considered a forerunner of this line of thought; it certainly was a stimulus to these authors in their way of thinking about dimension theory.

The preceding shows that as a corollary to this limit theorem, one has the result of Nagami that if a space $X$ is the limit of an inverse sequence of 
metrizable spaces $X_{i}$ and if for each $i \in \mathbb{N}$, $\operatorname{dim} X_{i} \leq n$, then $\operatorname{dim} X \leq n$. But it goes even further. Suppose that $G$ is an abelian group and for each $i \in \mathbb{N}, \operatorname{dim}_{G} X_{i} \leq n$; then $\operatorname{dim}_{G} X \leq n$. This is a new result which was known previously only for the groups $\mathbb{Z}$ and $\mathbb{Z} / p$ where $p$ is a prime number (see $[\mathbf{R S}]$ ). In $[\mathbf{K u}]$ a somewhat similar result is stated (see page 39 ) for the limit of an inverse system of metric spaces whenever the limit is strongly paracompact, but no proof is given and it is not clear which coefficient groups are meant. The limit theorem for separable metrizable spaces and countable complexes $K$ appears in $[\mathbf{C h}]$ where the author was able to extract the result from the proof of Proposition 2.1 of [Ol]. For the case of compact (not necessarily metrizable spaces), the theory is completely developed in $[\mathbf{R u}]$ where it is proved that the extension property is always preserved in the limit even when the systems are approximate inverse systems. Such a result for standard inverse systems can be found as Theorem 2.2 of [DR].

Finally, the authors want to thank Ivan Ivanšić for many stimulating discussions during the preparation of this material. His advice was extremely critical in helping us clarify the presentation of our result.

\section{Preliminaries.}

The term map will always mean continuous function. Whenever $K$ is a simplicial complex, then we shall bestow its polyhedron $|K|$ with the weak (CW) topology. Good references for the basics of simplicial complexes and their polyhedra with the weak topology are Appendix 1 of [MS] and Chapter 3 of $[\mathbf{S p}]$.

The notation $\operatorname{st}(v, K)$ will refer to the open star of the vertex $v$ of $K$. If $\mathcal{U}=\left\{U_{v} \mid v \in \Gamma\right\}$ is an (indexed) open cover of a space $X$, then $N(\mathcal{U})$ will denote the nerve of $\mathcal{U}$. Its vertex set consists of those $U_{v}$ which are not empty and hence the indexing set for the vertices of the nerve may well be a proper subset of $\Gamma$. On the other hand, it is sometimes convenient just to write $\left\{U_{v} \mid v \in \Gamma\right\}$ for the vertex set; one then understands that for some elements $v \in \Gamma, U_{v}$ need not be a vertex of the nerve.

When such an open cover is given, then a map $f: X \rightarrow|N(\mathcal{U})|$ is called $\mathcal{U}$-canonical if for each vertex $U_{v}$ of $N(\mathcal{U}), f^{-1}\left(\operatorname{st}\left(U_{v}, N(\mathcal{U})\right)\right) \subset U_{v}$. We want to emphasize that an (indexed) open collection $\mathcal{U}=\left\{U_{v} \mid v \in \Gamma\right\}$ is called locally finite if it is locally finite with respect to the indexing set $\Gamma$. This means that for each $x \in X$, there exists a neighborhood $V$ of $x$ in $X$ having the property that $V \cap U_{v} \neq \emptyset$ for at most finitely many $v \in \Gamma$.

For convenience to the reader, we are going to state here some results which can readily be deduced from other sources. The first is III.10.2 of $[\mathbf{H u}]$. (We shall use the notation $K^{(n)}$ to designate the $n$-skeleton of a given simplicial complex $K$.) 
Lemma 2.1. Let $X$ be a space satisfying the first countability axiom, $K$ be a simplicial complex, and $f: X \rightarrow|K|$ be a map. Then the (indexed) collection $\left\{Q_{v}=f^{-1}(\operatorname{st}(v, K)) \mid v \in K^{(0)}\right\}$ is a locally finite open cover of $X$.

The next one can be deduced easily from II.18.3 of $[\mathbf{H u}]$.

Lemma 2.2. Let $P$ be a closed subset of a metrizable space $B$ and $\mathcal{D}=$ $\left\{D_{v} \mid v \in \Gamma\right\}$ be a locally finite open cover of $P$ (by sets open in $P$ ). Then there exists a locally finite collection $\mathcal{D}^{*}=\left\{D_{v}^{*} \mid v \in \Gamma\right\}$ of open subsets of $B$ having the property that $D_{v}^{*} \cap P=D_{v}$ for each $v \in \Gamma$.

Notation 2.3. Let $\mathcal{Q}=\left\{Q_{v} \mid v \in \Gamma\right\}$ be a collection of sets. For each $v \in \Gamma$, let $\beta_{v}^{\mathcal{Q}}:|N(\mathcal{Q})| \rightarrow I$ denote the $Q_{v}$-barycentric coordinate function. Note of course that $\beta_{v}^{\mathcal{Q}}$ is positive on $\operatorname{st}\left(Q_{v}, N(\mathcal{Q})\right)$ and is zero elsewhere.

Lemma 2.4. Let $P$ be a closed subset of a metrizable space $B$ and $\mathcal{U}=$ $\left\{U_{v} \mid v \in \Gamma\right\}$ be an open cover of $B$. Put $\mathcal{E}=\left\{E_{v}=U_{v} \cap P \mid v \in \Gamma\right\}$ and let $f: P \rightarrow|N(\mathcal{E})|$ be an $\mathcal{E}$-canonical map. Let $\theta: N(\mathcal{E}) \rightarrow N(\mathcal{U})$ be the simplicial injection determined by the vertex map $E_{v} \mapsto U_{v}$. Then there is a $\mathcal{U}$-canonical map $g: B \rightarrow|N(\mathcal{U})|$ such that $g(P) \subset \theta(|N(\mathcal{E})|)$ and for all $x \in P, \theta^{-1}(g(x))=f(x)$ (thus, $\left.\theta(f(x))=g(x)\right)$.

Proof. Let $\Gamma_{0} \subset \Gamma$ consist of those $v$ such that $E_{v} \neq \emptyset$. For $v \in \Gamma_{0}$, let $D_{v}=f^{-1}\left(\operatorname{st}\left(E_{v}, N(\mathcal{E})\right)\right) \subset E_{v} \subset U_{v}$. By 2.1, $\left\{D_{v} \mid v \in \Gamma_{0}\right\}$ is a locally finite open cover of $P$. Using 2.2, find a locally finite collection $\left\{D_{v}^{*} \mid v \in \Gamma_{0}\right\}$ of open subsets of $B$ such that $D_{v}^{*} \cap P=D_{v}$ and $D_{v}^{*} \subset U_{v}$ for each $v \in \Gamma_{0}$.

For $v \in \Gamma_{0}$, select a map $\gamma_{v}: B \rightarrow I$ such that

(1) $\gamma_{v}=\beta_{v}^{\mathcal{E}} f$ on $P$, and

(2) $\gamma_{v}$ is zero on $B \backslash D_{v}^{*}$.

Choose a $\mathcal{U}$-canonical map $h: B \rightarrow|N(\mathcal{U})|$. Let $\Gamma_{1} \subset \Gamma$ consist of all $v$ such that $U_{v} \neq \emptyset$. Note that $\Gamma_{0} \subset \Gamma_{1}$ and that if $v \in \Gamma_{1} \backslash \Gamma_{0}$, then $U_{v} \cap P=\emptyset$. Choose a map $k: B \rightarrow I$ with the property that $k$ is zero on $P$ and is positive elsewhere. We define certain maps $\rho_{v}: B \rightarrow[0, \infty), v \in \Gamma_{1}$, in the following manner. Let $x \in B$; then,

(3) $\rho_{v}(x)=k(x) \beta_{v}^{\mathcal{U}} h(x)$ if $v \in \Gamma_{1} \backslash \Gamma_{0}$, and

(4) $\rho_{v}(x)=k(x) \beta_{v}^{\mathcal{U}} h(x)+\gamma_{v}(x)$ if $v \in \Gamma_{0}$.

We claim that each $x \in B$ has a neighborhood $T_{x}$ in $B$ on which $\rho_{v}$ is different from zero for only finitely many $v \in \Gamma_{1}$, and that $\rho_{v}(x)>0$ for at least one $v \in \Gamma_{1}$. To see the truth of the latter, first consider $x \in P$. Then for some $v \in \Gamma_{0}, f(x) \in \operatorname{st}\left(E_{v}, N(\mathcal{E})\right)$; from (1) we see that $\gamma_{v}(x)>0$. So (4) shows that $\rho_{v}(x)>0$. If $x \in B \backslash P$, then there has to be $v \in \Gamma_{1}$ such that $h(x) \in \operatorname{st}\left(U_{v}, N(\mathcal{U})\right)$. Now $k(x)>0$ and from the preceding one sees that $\beta_{v}^{\mathcal{U}} h(x)>0$. So whichever of (3) or (4) applies, we again conclude that $\rho_{v}(x)>0$. 
To find $T_{x}$, proceed as follows. Lemma 2.1 shows that $\left\{h^{-1}\left(\operatorname{st}\left(U_{v}\right.\right.\right.$, $\left.N(\mathcal{U}))) \mid v \in \Gamma_{1}\right\}$ is locally finite. Choose $T_{x}$ so that it intersects $h^{-1}\left(\operatorname{st}\left(U_{v}\right.\right.$, $N(\mathcal{U}))$ ) for only finitely many elements $v$ of $\Gamma_{1}$ and simultaneously that $T_{x}$ intersects $D_{v}^{*}$ for only finitely many $v \in \Gamma_{0}$. Let $w$ be an element of $\Gamma_{1}$ which is not one of these $v$ and $y \in T_{x}$. Now $y \notin h^{-1}\left(\operatorname{st}\left(U_{w}, N(\mathcal{U})\right)\right)$, so $h(y) \notin \operatorname{st}\left(U_{w}, N(\mathcal{U})\right)$. One sees from 2.2 that $\beta_{w}^{\mathcal{U}}(h(y))=0$. Since $y \notin D_{w}^{*}$, then by (2), $\gamma_{w}(y)=0$ if $w \in \Gamma_{0}$. It follows from (3) and (4) that $\rho_{w}(y)=0$.

We obtain a partition of unity $\left\{\rho_{v}^{*} \mid v \in \Gamma_{1}\right\}$ on $B$ from the preceding by setting

$$
\rho_{v}^{*}=\frac{\rho_{v}}{\sum\left\{\rho_{w} \mid w \in \Gamma_{1}\right\}} .
$$

The reader will not have difficulty (an argument similar to the one we just employed) seeing that each $\rho_{v}$ is zero outside $U_{v}$. Hence we may state that

(5) for all $v \in \Gamma_{1}, \rho_{v}^{*}$ is zero outside $U_{v}$.

From this it is seen that the formula

(6) $g(x)=\sum\left\{\rho_{v}^{*}(x) U_{v} \mid v \in \Gamma_{1}\right\}$

determines a function $g: B \rightarrow|N(\mathcal{U})|$. Since each $x \in B$ has a neighborhood on which $\rho_{v}$ (and hence $\rho_{v}^{*}$ ) is different from zero for only finitely many $v$, it is clear that $g$ is a map. For $x \in B \backslash U_{v}, \beta_{v}^{\mathcal{U}}(g(x))=0$ because of (5). Hence $g$ is a $\mathcal{U}$-canonical map.

Now suppose that $x \in P$. Let us show that if $v \in \Gamma_{1} \backslash \Gamma_{0}$, then $\beta_{v}^{\mathcal{U}}(g(x))=$ 0 . But the latter is just $\rho_{v}^{*}(x)$ which, by its definition, is a multiple of $\rho_{v}(x)$. The latter is zero because of (3) and the fact that $k(x)=0$. If at last we can show that for each $v \in \Gamma_{0}, \rho_{v}^{*}(x)=\beta_{v}^{\mathcal{E}}(f(x))$, then the rest of 2.4 will certainly be true. The denominator in the definition of $\rho_{v}^{*}(x)$ is nothing but $\sum\left\{\rho_{w}(x) \mid w \in \Gamma_{0}\right\}$. But since $k(x)=0$, this simplifies to $\sum\left\{\gamma_{w}(x) \mid w \in \Gamma_{0}\right\}=1$ (see (4) and (1)). The numerator is of course just $\gamma_{v}(x)=\beta_{v}^{\mathcal{E}}(f(x))$. Our proof is complete.

Lemma 2.5. Let $X$ be a metrizable space, $K$ be a simplicial complex, and $f, g: X \rightarrow|K|$ be maps such that for each $x \in X$, there is a simplex $\sigma$ of $K$ such that $f(x), g(x) \in \sigma$. Then $f \simeq g$.

Proof. Let $x \in X$. Applying 2.1, there is a neighborhood $V$ of $x$ and a finite subset $\mathcal{F}$ of $K^{(0)}$ such that $V \cap f^{-1}(\operatorname{st}(v, K))=\emptyset$ or $V \cap g^{-1}(\operatorname{st}(v, K))=\emptyset$ unless $v \in \mathcal{F}$. Let $L$ be the maximal finite subcomplex of $K$ whose vertex set is $\mathcal{F}$. Then the straight line homotopy between $f$ and $g$ using simplexes $\sigma$ as indicated in the hypothesis and restricted to $V$ has its image in $|L|$. Since the barycentric coordinates of the straight line homotopy are continuous and $L$ is finite, then this homotopy is continuous on $V$.

We need to develop some terminology. 
Definition 2.6. Let $f: X \rightarrow Y$ be a map and $W$ be an open subset of $X$. Then $\operatorname{resp}(W, f)$ is the maximal open subset $U$ of $Y$ such that $f^{-1}(U) \subset W$. We call $U$ the $W$-response to $f$. Suppose that $\mathcal{W}=\left\{W_{v} \mid v \in \Gamma\right\}$ is an indexed collection of open subsets of $X$. Then by $\operatorname{resp}(\mathcal{W}, f)$ we mean the (indexed) collection, $\left\{U_{v}=\operatorname{resp}\left(W_{v}, f\right) \mid v \in \Gamma\right\}$.

We ask the reader to fill in a proof of the next lemma.

Lemma 2.7. Let $W \subset W^{\prime}$ be open subsets of a space $X$, and let $f: X \rightarrow$ $Y, g: Y \rightarrow Z$ be maps. Write $h=g f: X \rightarrow Z$. Then $g^{-1}(\operatorname{resp}(W, h)) \subset$ $\operatorname{resp}(W, f) \subset \operatorname{resp}\left(W^{\prime}, f\right)$.

\section{The Limit Theorem.}

This section contains our main result.

Theorem 3.1. Let $K$ be a simplicial complex and $X=\lim \mathbf{X}$ where $\mathbf{X}=$ $\left(X_{i}, p_{i i+1}, \mathbb{N}\right)$ is an inverse sequence of metrizable spaces $X_{i}$ with $X_{i} \tau|K|$ for all $i \in \mathbb{N}$. Then $X \tau|K|$.

Proof. Note first that $X$ is a metrizable space. Let $A \subset X$ be closed and $f$ : $A \rightarrow|K|$ be a map. We have to prove that there exists a map $F: X \rightarrow|K|$ such that $F|A=f: A \rightarrow| K \mid$. We assume without loss of generality that $A \neq \emptyset$.

Extend $f$ to a map $f_{0}: W_{0} \rightarrow|K|$ where $W_{0}$ is an open neighborhood of $A$ in $X$. Let $W_{0}^{*}$ be an open neighborhood of $A$ in $X$ whose closure $\bar{W}_{0}^{*}$ (relative to $X$ ) is contained in $W_{0}$ and so that int $\bar{W}_{0}^{*}=W_{0}^{*}$.

For each $v \in K^{(0)}$, let $W_{v}^{0}=f_{0}^{-1}(\operatorname{st}(v, K))$. By Lemma $2.1, \mathcal{W}^{0}=$ $\left\{W_{v}^{0} \mid v \in K^{(0)}\right\}$ is a locally finite open cover of $W_{0}$ in terms of indexing by the set $K^{(0)}$. The identity function $K^{(0)} \rightarrow K^{(0)}$ induces a simplicial injection, $\eta_{0}: N\left(\mathcal{W}^{0}\right) \rightarrow K$ by sending the vertex $W_{v}^{0}$ to $v$.

We shall denote by $\Gamma$ the subset of $K^{(0)}$ consisting of those $v$ such that $W_{v}^{0} \cap \partial W_{0}^{*} \neq \emptyset$. There is a collection $\mathcal{W}^{1}=\left\{W_{v}^{1} \mid v \in \Gamma\right\}$ of open subsets of $W_{0}$ which is locally finite in $X$ and such that for each $v \in \Gamma, W_{v}^{1} \subset W_{v}^{0}$ and $W_{v}^{1} \cap \bar{W}_{0}^{*}=W_{v}^{0} \cap \bar{W}_{0}^{*}$. This is easily accomplished by applying Lemma 2.2. Let $W_{1}=\bigcup \mathcal{W}^{1}$; of course, $\mathcal{W}^{1}$ is an open cover of $\partial W_{0}^{*}$ in $W_{0}$. We define

(1) $f_{1}=f_{0}\left|W_{1}: W_{1} \rightarrow\right| K \mid$.

The inclusion $\Gamma \hookrightarrow K^{(0)}$ induces a simplicial injection of nerves, $\eta_{1}: N\left(\mathcal{W}^{1}\right)$ $\rightarrow N\left(\mathcal{W}^{0}\right)$ so that $\eta_{1}\left(W_{v}^{1}\right)=W_{v}^{0}$.

Let $H=X \backslash \bar{W}_{0}^{*}$; then $H$ is an open subset of $X$. Fix $i \in \mathbb{N}$. We want to name certain subsets and collections of subsets of $X_{i}$. First put

(2) $H_{i}=\operatorname{resp}\left(H, p_{i}\right)$.

Then of course,

(3) $H_{i}$ is open in $X_{i}, p_{i}^{-1}\left(H_{i}\right) \subset H$, and $H=\bigcup\left\{p_{i}^{-1}\left(H_{i}\right) \mid i \in \mathbb{N}\right\}$. Since $p_{i+1} p_{i+1}=p_{i}$, then Lemma 2.7 shows that, 
(4) $p_{i i+1}^{-1}\left(H_{i}\right) \subset H_{i+1}$.

Using a recursive construction on the index $i$, all the while applying (4), choose sequences $\left(H_{i}^{j}\right)_{j=1}^{\infty}$, each $\left(H_{i}^{j}\right)_{j=1}^{\infty}$ being a sequence of closed subsets of $X_{i}$, so that

(5) $H_{i}^{j} \subset H_{i}^{j+1} \subset H_{i}$ for each $j \in \mathbb{N}$,

(6) $\bigcup\left\{\operatorname{int} H_{i}^{j} \mid j \in \mathbb{N}\right\}=H_{i}$, and

(7) $p_{k i+1}^{-1}\left(H_{k}^{i+1}\right) \subset H_{i+1}^{1}$ whenever $1 \leq k \leq i$.

For each $v \in \Gamma$, define $U_{i, v}=\operatorname{resp}\left(W_{v}^{1}, p_{i}\right)$. We thus have a certain indexed open collection in $X_{i}: \mathcal{U}_{i}=\left\{U_{i, v} \mid v \in \Gamma\right\}$. This gives rise to an open subset of $X_{i}$, namely, $U_{i}=\bigcup \mathcal{U}_{i}$. Since $p_{i}^{-1}\left(U_{i, v}\right) \subset W_{v}^{1} \in \mathcal{W}^{1}$, the identity function $\Gamma \rightarrow \Gamma$ induces a simplicial injection $\beta_{i}: N\left(\mathcal{U}_{i}\right) \rightarrow N\left(\mathcal{W}^{1}\right)$ where $\beta_{i}\left(U_{i, v}\right)=W_{v}^{1}$. Taking into account Lemma 2.7 and the fact that $p_{i+k} p_{i+k}=p_{i}$, one deduces that for all $k \in \mathbb{N}$,

and, moreover,

(8) $p_{i i+k}^{-1}\left(U_{i, v}\right) \subset U_{i+k, v}$,

(9) $p_{i i+k}^{-1}\left(U_{i}\right) \subset U_{i+k}$.

We need to select some more closed sets in our space $X_{i}$. Let $Z_{i}=$ $\operatorname{cl}_{X_{i}}\left(p_{i}\left(\partial_{X} H\right)\right)$. Consider the open subset $K_{i}=U_{i} \cap Z_{i}$ of $Z_{i}$. Choose a sequence $\left(K_{i}^{j}\right)$ of closed subsets of $X_{i}$ such that

(10) $K_{i}^{j} \subset K_{i}^{j+1} \subset K_{i}$ for each $j \in \mathbb{N}$,

(11) $\bigcup\left\{K_{i}^{j} \mid j \in \mathbb{N}\right\}=K_{i}$, and

(12) $p_{k i+1}^{-1}\left(K_{k}^{i}\right) \cap Z_{i+1} \subset K_{i+1}^{1}$ whenever $1 \leq k \leq i$.

The latter is possible because of $(9)$.

From (3), $p_{i}^{-1}\left(H_{i}\right) \subset H$, so $Z_{i} \cap H_{i}=\emptyset$ and therefore $K_{i}^{1} \cap H_{i}^{1}=\emptyset$. We choose a closed neighborhood $D_{i}$ of $K_{i}^{1}$ in such a manner that

(13) $D_{i} \cap H_{i}^{1}=\emptyset$, and

(14) $D_{i} \subset U_{i}$.

Let us put

(15) $D_{i}^{*}=\bigcup\left\{p_{j i}^{-1}\left(D_{j}\right) \mid 1 \leq j \leq i\right\}$.

Then $D_{i}^{*}$ is a closed subset of $X_{i}$. Further, (15), (14) and (9) show that,

(16) $D_{i}^{*} \subset U_{i}$ and $D_{i+1}^{*}=p_{i i+1}^{-1}\left(D_{i}^{*}\right) \cup D_{i+1}$.

There is an indexed open cover designated $\mathcal{E}_{i}=\left\{E_{i, v}=U_{i, v} \cap D_{i}^{*} \mid v \in \Gamma\right\}$ of $D_{i}^{*}$. The vertex map $E_{i, v} \mapsto U_{i, v}$ determines a simplicial injection $\tau_{i}$ : $N\left(\mathcal{E}_{i}\right) \rightarrow N\left(\mathcal{U}_{i}\right)$.

We define,

(17) $\alpha_{i}=\eta_{0} \eta_{1} \beta_{i} \tau_{i}: N\left(\mathcal{E}_{i}\right) \rightarrow K$,

and note that $\alpha_{i}$ is a simplicial injection.

On the other hand, suppose that $E_{i, v}$ is a vertex of $N\left(\mathcal{E}_{i}\right)$, i.e., $E_{i, v} \neq$ $\emptyset$. Using (8) and (16), one can see that $E_{i+1, v} \neq \emptyset$ and that, indeed, $p_{i i+1}^{-1}\left(E_{i, v}\right) \subset E_{i+1, v}$. Hence the vertex map, $E_{i, v} \mapsto E_{i+1, v}$ determines a 
simplicial injection $\theta_{i}: N\left(\mathcal{E}_{i}\right) \rightarrow N\left(\mathcal{E}_{i+1}\right)$. Of course (8) shows that the vertex map $U_{i, v} \mapsto U_{i+1, v}$ (whenever $U_{i, v} \neq \emptyset$ ) determines a simplicial injection $\theta_{i}^{*}: N\left(\mathcal{U}_{i}\right) \rightarrow N\left(\mathcal{U}_{i+1}\right)$, and one can see from the definitions that,

(18) $\theta_{i}^{*} \tau_{i}=\tau_{i+1} \theta_{i}$ and $\beta_{i+1} \theta_{i}^{*}=\beta_{i}$.

Now for the first step of an inductive procedure. Choose an $\mathcal{E}_{1}$-canonical $\operatorname{map} g_{1}: D_{1}^{*} \rightarrow\left|N\left(\mathcal{E}_{1}\right)\right|$. Since $X_{1} \tau|K|, \alpha_{1} g_{1}: D_{1}^{*} \rightarrow|K|$ is a map, and $D_{1}^{*}$ is closed in $X_{1}$, then there is a map $g_{1}^{*}: D_{1}^{*} \cup H_{1}^{1} \rightarrow|K|$ which is an extension of $\alpha_{1} g_{1}$.

Let $k \in \mathbb{N}$. Assume that for each $1 \leq i \leq k$ we have defined:

(I1) an $\mathcal{E}_{i}$-canonical map $g_{i}: D_{i}^{*} \rightarrow\left|N\left(\mathcal{E}_{i}\right)\right|$, and

(I2) a map $g_{i}^{*}: D_{i}^{*} \cup H_{i}^{1} \rightarrow|K|$ which is an extension of $\alpha_{i} g_{i}: D_{i}^{*} \rightarrow$ $|K|$.

We assume that this has been done so that if $1<i \leq k$, then

(I3) $g_{i}^{*}(x)=g_{i-1}^{*} p_{i-1 i}(x)$ for all $x \in p_{i-1 i}^{-1}\left(D_{i-1}^{*} \cup H_{i-1}^{1}\right)$.

Let $P=p_{k k+1}^{-1}\left(D_{k}^{*}\right)$ and put $\mathcal{E}=\left\{E_{v}=E_{k+1, v} \cap P \mid v \in \Gamma\right\}$. (Use (16) and the fact that $\mathcal{E}_{k+1}$ is an open cover of $D_{k+1}^{*}$.) For each vertex $E_{k, v}$ of $N\left(\mathcal{E}_{k}\right)$, we know that $p_{k k+1}^{-1}\left(E_{k, v}\right) \subset E_{k+1, v}$. From its definition, $E_{k, v} \subset D_{k}^{*}$. Using (16),

(19) $p_{k k+1}^{-1}\left(E_{k, v}\right) \subset E_{k+1, v} \cap P=E_{v}$;

so the vertex map $E_{k, v} \mapsto E_{v}$ determines a simplicial injection $\phi: N\left(\mathcal{E}_{k}\right) \rightarrow$ $N(\mathcal{E})$. Define $\hat{f}: P \rightarrow|N(\mathcal{E})|$ by

(20) $\hat{f}(x)=\phi g_{k} p_{k k+1}(x), x \in P=p_{k k+1}^{-1}\left(D_{k}^{*}\right)$.

We wish to show that

(21) $\hat{f}$ is an $\mathcal{E}$-canonical map.

Surely $\phi^{-1}\left(\operatorname{st}\left(E_{v}, N(\mathcal{E})\right)\right) \subset \operatorname{st}\left(E_{k, v}, N\left(\mathcal{E}_{k}\right)\right)$ for each vertex $E_{v}$ of $N(\mathcal{E})$. From (I1) we get that $g_{k}^{-1}\left(\operatorname{st}\left(E_{k, v}, N\left(\mathcal{E}_{k}\right)\right)\right) \subset E_{k, v}$. We conclude from this, (20), and (19) that (21) is true.

Next define $\theta: N(\mathcal{E}) \rightarrow N\left(\mathcal{E}_{k+1}\right)$ to be the simplicial injection determined by the vertex map $E_{v} \mapsto E_{k+1, v}$. Let $B=D_{k+1}^{*}$. Then with $\hat{f}$ in place of $f$ and $\mathcal{E}_{k+1}$ in place of $\mathcal{U}$, we may apply Lemma 2.4. This yields an $\mathcal{E}_{k+1^{-}}$ canonical map $g_{k+1}: D_{k+1}^{*} \rightarrow\left|N\left(\mathcal{E}_{k+1}\right)\right|$ as requested in (I1), having the property that for $x \in p_{k k+1}^{-1}\left(D_{k}^{*}\right), \theta \hat{f}(x)=g_{k+1}(x)$. So (20) shows that $g_{k+1}(x)=\theta \phi g_{k} p_{k+1}(x)$. One readily checks that $\theta \phi=\theta_{k}$, so

(22) $g_{k+1}(x)=\theta_{k} g_{k} p_{k k+1}(x), x \in p_{k k+1}^{-1}\left(D_{k}^{*}\right)$.

Now $\alpha_{k+1} g_{k+1}(x) \in|K|$ and by the definition of $\alpha_{k+1}$ and (18), $\alpha_{k+1} \theta_{k}=$ $\eta_{0} \eta_{1} \beta_{k+1} \tau_{k+1} \theta_{k}=\eta_{0} \eta_{1} \beta_{k+1} \theta_{k}^{*} \tau_{k}=\eta_{0} \eta_{1} \beta_{k} \tau_{k}$. From this and (22), $\alpha_{k+1} g_{k+1}(x)=\eta_{0} \eta_{1} \beta_{k} \tau_{k} g_{k} p_{k k+1}(x)=\alpha_{k} g_{k} p_{k k+1}(x)$. Since $p_{k k+1}(x) \in D_{k}^{*}$, then (I2) shows that $\alpha_{k+1} g_{k+1}(x)=g_{k}^{*} p_{k k+1}(x)$. Therefore we may extend $\alpha_{k+1} g_{k+1}: p_{k k+1}^{-1}\left(D_{k}^{*}\right) \rightarrow|K|$ to a map $\hat{g}_{k+1}: p_{k k+1}^{-1}\left(D_{k}^{*} \cup H_{k}^{1}\right) \rightarrow|K|$ by setting

(23) $\hat{g}_{k+1}(x)=g_{k}^{*} p_{k k+1}(x), x \in p_{k k+1}^{-1}\left(D_{k}^{*} \cup H_{k}^{1}\right)$. 
Since $X_{k+1} \tau|K|$, we may extend $\hat{g}_{k+1}$ to a map $\tilde{g}_{k+1}: p_{k k+1}^{-1}\left(D_{k}^{*} \cup H_{k}^{1}\right) \cup$ $H_{k+1}^{1} \rightarrow|K|$. From (16) one sees that $D_{k+1}^{*}=p_{k k+1}^{-1}\left(D_{k}^{*}\right) \cup D_{k+1}$, and from (13) that $D_{k+1} \cap H_{k+1}^{1}=\emptyset$. Moreover, (7) shows that $p_{k k+1}^{-1}\left(H_{k}^{1}\right) \subset$ $H_{k+1}^{1}$. So $C=D_{k+1} \cap\left(p_{k k+1}^{-1}\left(D_{k}^{*} \cup H_{k}^{1}\right) \cup H_{k+1}^{1}\right)=D_{k+1} \cap\left(p_{k k+1}^{-1}\left(D_{k}^{*}\right) \cup\right.$ $\left.H_{k+1}^{1}\right) \subset p_{k k+1}^{-1}\left(D_{k}^{*}\right)$. On $C$ the map $\tilde{g}_{k+1}$ is defined by $\tilde{g}_{k+1}(x)=\hat{g}_{k+1}(x)=$ $\alpha_{k+1} g_{k+1}(x)$. We therefore extend $\tilde{g}_{k+1}$ to a map $g_{k+1}^{*}: p_{k k+1}^{-1}\left(D_{k}^{*} \cup H_{k}^{1}\right) \cup$ $H_{k+1}^{1} \cup D_{k+1}=D_{k+1}^{*} \cup H_{k+1}^{1} \rightarrow|K|$ by setting $g_{k+1}^{*}(x)=\alpha_{k+1} g_{k+1}(x)$, $x \in D_{k+1}$.

To check that (I2) is satisfied, consider $x \in D_{k+1}^{*}$. If $x \in D_{k+1}$, then we have just seen that $g_{k+1}^{*}(x)=\alpha_{k+1} g_{k+1}(x)$. If $x \in p_{k k+1}^{-1}\left(D_{k}^{*}\right)$, then $g_{k+1}^{*}(x)=\tilde{g}_{k+1}(x)$ which equals $\hat{g}_{k+1}(x)$ from $(23)$, since $\tilde{g}_{k+1}$ is an extension of $\hat{g}_{k+1}$. But, $\hat{g}_{k+1}$ is an extension of $\alpha_{k+1} g_{k+1}$ on $p_{k k+1}^{-1}\left(D_{k}^{*}\right)$. Finally, (I3) is manifest from (23). Our inductive construction is complete.

Claim. The preceding data uniquely determines a map $G: \bar{H} \rightarrow|K|$ such that $G\left|\partial_{X} H \simeq f_{0}\right| \partial_{X} H$.

Here is our justification of this claim. Let $x \in \bar{H}$. We shall define a neighborhood $M_{x}$ of $x$ in $\bar{H}$ and a map $G_{x}: M_{x} \rightarrow|K|$. Then we will observe that these maps $G_{x}$ agree on overlaps, and will put $G=\bigcup\left\{G_{x} \mid x \in \bar{H}\right\}$. We shall also see that $G$ has the desired property with regard to $\partial_{X} H$.

Consider first the case that $x \in H$. By (3), there exists a first $i$ such that $x \in p_{i}^{-1}\left(H_{i}\right)$. Subsequently (6) yields that there is a first $j$ with $x_{i} \in \operatorname{int}_{X_{i}} H_{i}^{j}$. Using (5), (7), there is a first $k=k(x) \geq i$ such that $p_{i k(x)}^{-1}\left(H_{i}^{j}\right) \subset H_{k(x)}^{1}$. Now $x_{k(x)} \in \operatorname{int}_{X_{k(x)}} p_{i k(x)}^{-1}\left(H_{i}^{j}\right)$, so $x \in \operatorname{int}_{\bar{H}} M_{x}$ where $M_{x}=p_{k(x)}^{-1}\left(p_{i k(x)}^{-1}\left(H_{i}^{j}\right)\right)=p_{i}^{-1}\left(H_{i}^{j}\right) \subset H$ by (3). Define $G_{x}: M_{x} \rightarrow|K|$ by $G_{x}(y)=g_{k(x)}^{*}\left(p_{k(x)}(y)\right)=g_{k(x)}^{*}\left(y_{k(x)}\right)$.

Now look at the other case, $x \in \partial_{X} H$. (Note that $\partial_{X} H=\partial_{X} W_{0}^{*}$.) There exists a neighborhood $V_{x}$ of $x$ in $W_{1}$ and a finite subset $\mathcal{F}_{x} \subset \Gamma$ such that $V_{x} \cap W_{v}^{1}=\emptyset$ unless $v \in \mathcal{F}_{x}$. We may as well assume that $V_{x} \subset W_{v}^{1}$ when $v \in \mathcal{F}_{x}$. There exists a first $i$ and a neighborhood $V_{x_{i}}$ of $x_{i}$ in $X_{i}$ such that $x \in p_{i}^{-1}\left(V_{x_{i}}\right) \subset V_{x}$. Choose $v \in \mathcal{F}_{x}$; then $x_{i} \in V_{x_{i}} \subset U_{i, v}=\operatorname{resp}\left(W_{v}^{1}, p_{i}\right) \subset$ $U_{i}$.

Hence, $x_{i} \in K_{i}=U_{i} \cap Z_{i}$. So there exists a first $j$ with $x_{i} \in K_{i}^{j}$. Apply (12) and see that there exists a first $k=k(x) \geq i$ with $x_{k(x)} \in K_{k(x)}^{1}$. Recall that $D_{k(x)}$ is a neighborhood of $K_{k(x)}^{1}$ in $X_{k(x)}$ and that $D_{k(x)} \subset D_{k(x)}^{*}$. Define $M_{x}=p_{k(x)}^{-1}\left(D_{k(x)}^{*}\right) \cap \bar{H}$ and note that $x \in \operatorname{int}_{\bar{H}} M_{x}$. Define $G_{x}$ : $M_{x} \rightarrow|K|$ by $G_{x}(y)=g_{k(x)}^{*} p_{k(x)}(y)=g_{k(x)}^{*}\left(y_{k(x)}\right)$. 
Each $G_{x}$ is a map, and $\left\{\operatorname{int}_{\bar{H}} M_{x} \mid x \in \bar{H}\right\}$ is an open cover of $\bar{H}$. If we can show that whenever $x, z \in \bar{H}$ and $y \in M_{x} \cap M_{z}$, then $G_{x}(y)=G_{z}(y)$, then these maps uniquely define a map of $\bar{H}$ to $|K|$.

To see this, assume without loss of generality that $k(x) \leq k(z)$. Indeed, we may as well assume that $k(x)<k(z)$, for if they were equal, then $G_{x}$ and $G_{z}$ would have been defined at $y$ by the same formula. Note that $G_{z}(y)=g_{k(z)}^{*}\left(y_{k(z)}\right)$.

First suppose that $M_{x}=p_{k(x)}^{-1}\left(D_{k(x)}^{*}\right) \cap \bar{H}$, i.e., $x \in \partial H$. Then

$$
\text { (24) } y_{k(z)} \in p_{k(x) k(z)}^{-1}\left(D_{k(x)}^{*}\right) \text {. }
$$

An application of (24) and (I3) shows that

$$
G_{z}(y)=g_{k(z)}^{*}\left(y_{k(z)}\right)=g_{k(x)}^{*} p_{k(x) k(z)}\left(y_{k(z)}\right)=g_{k(x)}^{*}\left(y_{k(x)}\right)=G_{x}(y) .
$$

Alternatively, $M_{x}=p_{i}^{-1}\left(H_{i}^{j}\right)$ where $k(x) \geq i$ and $x_{i} \in \operatorname{int}_{X_{i}} H_{i}^{j}$. Moreover, $p_{i k(x)}^{-1}\left(H_{i}^{j}\right) \subset H_{k(x)}^{1}$. This shows that

$$
\text { (25) } y_{k(z)} \in p_{k(x) k(z)}^{-1}\left(H_{k(x)}^{1}\right) \text {. }
$$

Just apply (25) and (I3) to see that $G_{z}(y)=G_{x}(y)$ as in the previous situation.

We are now assured that $G$ is a well-defined map. To complete the proof of the claim, suppose that $x \in \partial_{X} H$. Then $x \in M_{x} \subset p_{k(x)}^{-1}\left(D_{k(x)}^{*}\right)$ and we have defined $G(x)=G_{x}(x)=g_{k(x)}^{*} p_{k(x)}(x)$. Now $p_{k(x)}(x) \in D_{k(x)}^{*}$; by applying (I2), we see that $G(x)=\alpha_{k(x)} g_{k(x)} p_{k(x)}(x)=\alpha_{k(x)} g_{k(x)}\left(x_{k(x)}\right)$.

Let us note that if $x_{k(x)} \in U_{k(x), v}$, then it has to be true that $v \in \mathcal{F}_{x}$. To see this, note that $x_{k(x)} \in p_{i k(x)}^{-1}\left(V_{x_{i}}\right)$, and hence $x \in p_{k(x)}^{-1} p_{i k(x)}^{-1}\left(V_{x_{i}}\right)=$ $p_{i}^{-1}\left(V_{x_{i}}\right) \subset V_{x}$. Moreover, $x_{k(x)} \in p_{i k(x)}^{-1}\left(V_{x_{i}}\right) \cap U_{k(x), v}$, and since $U_{k(x), v}=$ $\operatorname{resp}\left(W_{v}^{1}, p_{k(x)}\right)$, then $x \in p_{k(x)}^{-1}\left(U_{k(x), v}\right) \subset W_{v}^{1}$. Therefore $x \in V_{x} \cap W_{v}^{1}$, so $v \in \mathcal{F}_{x}$ as stated.

By (I1), $g_{k(x)}$ is an $\mathcal{E}_{k(x)^{-}}$-canonical map. So for some subset $\mathcal{F}_{k(x)} \subset \mathcal{F}_{x}$, $g_{k(x)}\left(x_{k(x)}\right)$ lies in the simplex whose vertices are $\left\{U_{k(x), v} \mid v \in \mathcal{F}_{k(x)}\right\}$. The map $\alpha_{k(x)}$ sends $g_{k(x)}\left(x_{k(x)}\right)$ into the simplex of $K$ having vertices $\left\{W_{v}^{1} \mid v \in\right.$ $\left.\mathcal{F}_{k(x)}\right\} \subset\left\{W_{v}^{1} \mid v \in \mathcal{F}_{x}\right\}$.

But the map $f_{0}$ sends $x$ into the simplex of $K$ having vertices $\left\{W_{v}^{1} \mid v \in\right.$ $\left.\mathcal{F}_{x}\right\}$. Hence by Lemma 2.5, $G\left|\partial_{X} H \simeq f_{0}\right| \partial_{X} H$. Our proof of Theorem 3.1 is completed by an application of the homotopy extension theorem.

Corollary 3.2. Let $X=\lim \mathbf{X}$ where $\mathbf{X}=\left(X_{i}, p_{i i+1}, \mathbb{N}\right)$ is an inverse sequence of metrizable spaces $X_{i}$. Suppose that $\operatorname{dim} X_{i} \leq n(G$ is an abelian group and $\left.\operatorname{dim}_{G} X_{i} \leq n\right)$ for all $i \in \mathbb{N}$. Then $\operatorname{dim} X \leq n\left(\operatorname{dim}_{G} X \leq n\right)$.

Let us remark that a proof of this result for dim can be found in $[\mathrm{Na}]$. 


\section{References}

[Ch] A. Chigogidze, Cohomological dimension of Tychonov spaces, Topology and its Appls., 79 (1997), 197-228.

[DR] T. Dobrowolski and L. Rubin, The hyperspaces of infinite-dimensional compacta for covering and cohomological dimension are homeomorphic, Pacific J. of Math., 164 (1994), 15-39.

[DD] A. Dranishnikov and J. Dydak, Extension theory of separable metrizable spaces with applications to dimension theory (preliminary version), preprint.

[Hu] S. Hu, Theory of retracts, Wayne State University Press, Detroit, 1965.

[Ku] W.I. Kuz'minov, Homological dimension theory, Russian Math. Surveys, 23 (1968), $1-45$.

[MS] S. Mardešić and J. Segal, Shape theory, North-Holland, Amsterdam, 1982.

[Na] K. Nagami, Dimension theory, Academic Press, New York, 1970.

[Ol] W. Olszewski, Universal separable metrizable spaces of given cohomological dimension, Topology and its Appls., 61 (1995), 293-299.

[Ru] L. Rubin, Cohomological dimension and approximate limits, Proc. Amer. Math. Soc., 125 (1997), 3125-3128.

[RS] L. Rubin and P. Schapiro, Some limit theorems for cohomological dimension in metric spaces, preprint.

[Sp] E. Spanier, Algebraic topology, McGraw-Hill, New York, 1966.

[Wa] J. Walsh, Dimension, cohomological dimension, and Cell-like mappings, in 'Shape Theory and Geometric Topology', Lecture Notes in Mathematics, Springer Verlag, Berlin, (1981), 105-118.

Received March 3, 1997

The University OF OKLAHOMA

NORMAN, OK 73019

E-mail address: lrubin@ou.edu

LANGSTON UNIVERSITY

LANGSTON, OK 73050

E-mail address: pjschapiro@lunet.edu 